\section{Isolated sarcoid myopathy: an unusual presentation}

\author{
Jayeeta Bhowmick, ${ }^{1}$ \\ Pramila Dharmshaktu, ${ }^{1}$ \\ Subhro Chakraborty ${ }^{2}$
}

1Department of Medicine, Maulana Azad Medical College, New Delhi; ${ }^{2}$ Department of Cardiology, R.G Kar Medical College, Kolkata, India

\section{Abstract}

Sarcoidosis is a systemic disease with protean clinical manifestations, pathologically characterized by the development of a noncaseating granuloma. Any organ system may be involved during the course of the disease with lung, liver, skin and eyes being commonly involved. Muscular sarcoidosis is a rare entity, seen in approximately $6 \%$ of patients with sarcoidosis. Symptomatic muscle involvement is even rarer, noted in less than $0.5 \%$ of patients. We report the case of a young male patient (14 years old), who presented to our hospital with unilateral hypertrophy of shoulder and calf muscles. After excluding all the possible causes, it was surprisingly diagnosed to be a case of muscle involvement with sarcoidosis. Patient also improved significantly with oral corticosteroid therapy. This report therefore highlights the importance of keeping this rare cause in mind when the common etiologies are excluded in a patient with hypertrophy of muscle. It may be rewarding if diagnosis is established early.

\section{Introduction}

Sarcoidosis is a multi-system inflammatory disorder of unknown cause. Most patients with this disease present with respiratory symptoms since lungs are frequently affected organs. However, any organ system may be involved in the course of this disease; liver, skin and eyes are as well commonly involved. 1,2

Symptomatic muscle involvement is rare and presentation of this disease as myopathy is even rarer. $^{3-5}$ The usual presentation of those with symptomatic disease is severe disabling proximal muscle weakness and pain as in this patient. Most patients are less than 40 years old. These patients may also present with diffuse muscular atrophy or tumor like growth. There are four types of muscular sarcoidosis: nodular, chronic myopathy, acute myositis, and asymptomatic. ${ }^{3,6,7}$ The acute myositic type occurs in the early stage of sarcoidosis, in which the inflammatory process causes myalgia. Magnetic resonance imaging (MRI) is unusually negative due to small size of lesions. ${ }^{8}$ Patient with chronic myopathic type present with myalgia, weakness and wasting of the muscles. MRI suggests nonspecific muscle atrophy. ${ }^{8}$ Muscle involvement in sarcoidosis is reported in many case reports. ${ }^{7,9}$ Finally the palpable nodular type is exceedingly rare, which is important because it may be confused with a soft tissue tumor. ${ }^{10}$

We report one patient of sarcoid myopathy presented with hypertrophy of left calf and shoulder muscle as the only presentation.

\section{Case Report}

A 14 year old boy, student, born out of non consanguineous marriage presented with gradual onset swelling of left calf and shoulder without any definite muscular weakness, pain, joint swelling, rash, oral ulcer, photophobia, easy fatigability, convulsion, loss of consciousness, altered mental status, symptoms of raised intracranial tension, cough, shortness of breath, chest pain, hemoptysis, hematuria, pain abdomen, neuropsychiatric manifestations, jaundice, bladder or bowel involvement, sensory or gait abnormality. On examination, patient had stable vitals with normal higher mental function, normal neurological examination except there was hypertrophy of left deltoid (Figure 1A) and left calf (Figure 1B) muscles and power of all four limbs were grade $4 / 5$ according to the Medical Research Council Power Grading System. All other systemic examinations are within normal limit.

Routine complete blood count, liver and kidney function tests, Serum Electrolytes, thyroid function, routine urine and stool examinations were within normal range. Blood test for VDRL, $\mathrm{HIV}, \mathrm{HBsAg}$ and HCV were non reactive. ANA, c-ANCA and p-ANCA were negative. Patient had a history of congenital acyanotic heart disease in the form of Ostium Primum type Atrial Septal Defect due to Endocardial cushion defect, diagnosed and operated upon at the age of 7 years with a short episode of wound infection and dehiscence followed by an uneventful recovery. Since then patient is doing well without any complaint regarding the heart disease.

No KF (Kayser Fleisher) ring was found on slit lamp examination of eye. Chest X-ray, HRCT Thorax and USG of whole abdomen revealed no abnormality. Fibreoptic bronchoscopy and bronchoalveolar lavage was normal. Upper G.I. endoscopy and colonoscopy were within normal limit. Laboratory investigation reports other than the routines in this case are reported in Table 1.

Musculoskeletal ultrasonography of deltoid and calf muscle showed grossly bulky muscle
Correspondence: Pramila Dharmshaktu, Department of Medicine, Maulana Azad Medical College, Bahadur Shah Zafar Marg, New Delhi, 110002, India.

Tel. +91.958.2934837.

E-mail: dr.pramila09@gmail.com

Key words: sarcoidosis, myopathy, sarcoid myopathy, muscle hypertrophy, non-caseating granuloma.

Acknowledgements: the authors would extend their acknowledgements to $\mathrm{Dr}$ Sayantan Banerjee, Department of Microbiology, Maulana Azad Medical College, New Delhi, India, for his generous academic and technical support.

Contributions: the authors contributed equally.

Conflict of interests: the authors declare no potential conflict of interests.

Received for publication: 12 August 2013.

Revision received: 7 January 2014.

Accepted for publication: 24 March 2014.

This work is licensed under a Creative Commons Attribution NonCommercial 3.0 License (CC BYNC 3.0).

CCopyright J. Bhowmick et al., 2014

Licensee PAGEPress, Italy

Rheumatology Reports 2014; 6:5024

doi:10.4081/rr.2014.5024

without any tear, fluid collection or calcification. Nerve conduction study was normal with electromyography showing generalized myopathic pattern. Muscle biopsy taken from calf muscle showing (Figure 2) polyfocal myonecrosis, focal endomysial interstitial inflammation, focal epitheloid cell granuloma (non caseating) with few perivascular inflammatory infiltrate suggestive of sarcoid myopathy. The patient was prescribed oral corticosteroid (prednisolone tablets $40 \mathrm{mg} /$ day) and after two months, there was objectively reduced muscle bulk clinically as well as evidenced by an ultrasonogram.

\section{Discussion and Conclusions}

This is a case of isolated sarcoid myopathy without prior or concurrent organ involvement. On follow up, there are no feature of other organ involvement on examining him clinically and on the basis of laboratory test like ESR, Liver function test and seum Calcium, phosphorus which were within normal range. As lung is the commonest organ to be involved in this disease we got a follow up CECT chest done which showed normal study. 

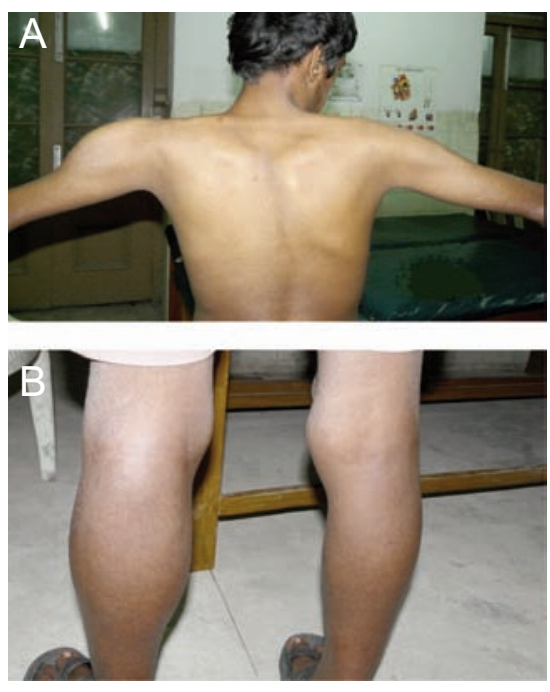

Figure 1. A picture from back showing left sided deltoid hypertrophy (A) and left sided calf hypertrophy (B).

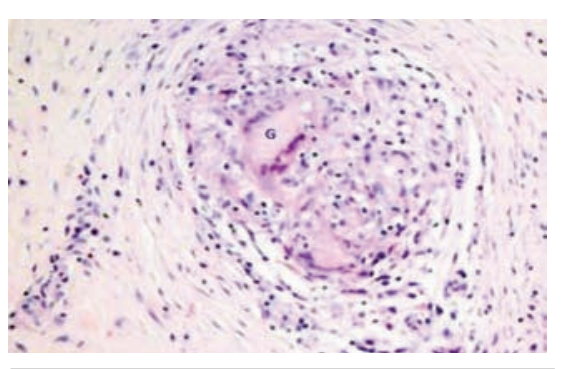

Figure 2. Histopathological photomicrograph of muscle biopsy showing noncaseating granuloma with a multinucleate giant cell $(G)$ with interstitial and perivascular infiltrate.

The diagnosis of myopathy in the said patient was entertained on the basis of clinical suspicion and electrophysiological test. We tried to exclude all possible causes of muscle hypertrophy like tuberculosis, inflammatory myopathy, cysticercosis, other groups of myopathy, malig-

Table 1. Laboratory findings.

\begin{tabular}{lcc} 
Investigation & Reference value & Patient's value \\
Serum lactate $(\mathrm{mg} / \mathrm{dL})$ & $4.5-20$ & 7.0 \\
Mantoux induration $(\mathrm{mm})$ & $<5.0$ & 4.0 \\
\hline CPK (U/L) & $25-200$ & 37 \\
ESR (mm/hr) & $<10$ & 62 \\
\hline Calcium (mg/dL) & $9-11$ & 14 \\
Serum albumin (g/dL) & & 4.5 \\
\hline Serum angiotensin converting enzyme (U/L) & $7-46$ & 64 \\
Thyroid stimulating hormone ( IU/mL) & $0.34-4.25$ & 2.19 \\
\hline
\end{tabular}

nancy or Paraneoplastic and thyroid related disorders by relevant investigations. However after muscle biopsy report, it was surprisingly found to be a case consistent with sarcoid myopathy. The interesting features in this patient was hypertrophy of isolated muscle like calf group and deltoid on left side due to sarcoidosis as the sole presenting symptoms without gross weakness, myalgia, focal neurological deficit or any other system involvement. So, it is a case of isolated sarcoid myopathy which is a very rare entity. The literatures show scanty report on such presentation. ${ }^{7,9}$ Hence, this case is presented to emphasize the fact that in any case of hypertrophy of muscle, when common causes are excluded, one should keep in mind the rare presentation of sarcoid myopathy which if treated early may give gratifying result. Our patient responded on oral steroid and he is under regular follow up till date.

\section{References}

1. Otake S, Imagumbai N, Suzuki M, Ohba S. MR imaging of muscular sarcoidosis after steroid therapy. Eur Radiol 1998;8:1651-3.

2. Heckmann JG, Stefan H, Heuss D, et al.
Isolated muscle sarcoidosis. Eur J Neurol 2001;8:365-6.

3. Kobayashi H, Kotoura Y, Sakahara H, et al. Solitary muscular sarcoidosis: CT, MRI and scintigraphic characteristic. Skeletal Radiol 1994;23:293-5.

4. Zisman D, Biermann JS, Martiniz F, et al. Sarcoidosis presenting as a tumor-like muscular lesion: case report and review of the literature. Medicine 1999;78:112-22.

5. Silverstein A, Siltzbach LE. Muscle involvement in sarcoidosis: asymptomatic, myositis and myopathy. Arch Neurol 1969;21: $235-41$.

6. Douglas AC, MacLeod JG, Mathew JD. Symptomatic sarcoidosis of skeletal muscle. J Neurol Neurosurg Psychiatry 1973; 36:1034-40.

7. Saharan S, Majithia V. Isolated muscle mass as an initial presentation of sarcoidosis: a case report and discussion. J Miss State Med Assoc 2008;49:107-9.

8. Otake S, Banno T, Ohba S, et al. Muscular sarcoidosis: findings at MR imaging. Radiology 1990;176:145-8.

9. Berger C, Sommer C, Meinck HM. Isolated sarcoid myopathy. Muscle Nerve 2002;26: 553-6.

10. Otake $\mathrm{S}$, Ishigaki T. Muscular sarcoidosis. Semin Musculoskelet Radiol 2001;5:167-70. 